\title{
Bose-Einstein Condensation of Hard Sphere Homogeneous Gas in Static Fluctuation Approximation
}

\author{
M.K. Al-Sugheir*, S.S. Gasymeh, M. Shatnawi and M.S. Bawa'Aneh \\ Department of Physics, Faculty of Science, The Hashemite University, Zarqa, Jordan
}

(Received April 22, 2009; in final form July 9, 2009)

\begin{abstract}
The condensation fraction, transition temperature, and energy per particle for a hard sphere interacting homogeneous Bose gas using the static fluctuation approximation have been determined. The transition temperature at liquid helium density has been found to be lower than that for the noninteracting gas. Both superfluidity and the Bose-Einstein condensation have been found to occur at the same transition temperature. Our results are consistent with results obtained by other methods.
\end{abstract}

PACS numbers: 03.75.Nt, 05.30.Jp, 67.40.--w, 67.25.D-, 67.25.de

\section{Introduction}

The observations of the Bose-Einstein condensation (BEC) in alkaline atoms motivate us to shed further light on homogeneous Bose gas using a hard sphere potential model. The simple hard sphere potential model is appropriate for a dilute Bose gas and reasonable for relatively dense systems [1]. Experimental developments of BEC in alkali vapors have stimulated a great interest in the theoretical studies of Bose gases. Specifically, in the presence of harmonic confinement, the many-body theory of interacting Bose gases has given rise to several unexpected features. This has opened new theoretical prospects in this field.

The validity of the static fluctuation approximation (SFA) [2-4] for arbitrary systems (strongly or weakly interacting systems) and at any finite temperature gives us the opportunity to study Bose gases at different potential strengths and ranges. The dynamics of weakly interacting Bose gases is modeled by the mean-field theories [5]. The SFA technique is more advanced compared with the mean field approximation, where in SFA, the square of the local-field operator is replaced by its mean value. The physical implication is that the true quantum-mechanical spectrum of this operator is replaced by a distribution around the expectation value of the local-field operator.

In many previous works the transition temperature was found to be dependent on the system density as well as on the interaction potential. The transition temperature for a repulsive interaction increased at low densities and decreased at high densities [6]. Different analytical formulae are observed for transition temperature,

\footnotetext{
* corresponding author; e-mail: msugh@hu.edu.jo
}

where in Ref. [7] the deviation in the transition temperature $T_{\mathrm{C}}$ for interacting Bose gas from that for ideal Bose gas $T_{0}$ is $\Delta T_{\mathrm{C}} / T_{0} \propto\left(n a^{3}\right)^{1 / 2}$, while Stoof has obtained $\Delta T_{\mathrm{C}} / T_{0} \propto\left(n a^{3}\right)^{1 / 3}$ in [8], and $\Delta T_{\mathrm{C}} / T_{0} \propto\left(n a^{3}\right)^{0.34}$ have obtained in [6], where $n$ is the density number of the system and $a$ is the $s$-wave scattering length. This discrepancy in the transition temperature in addition to the wide variety in calculated or measured condensate fraction and its dependence on the methods used [9-11] motivates us to shed more light on bosonic systems.

We will determine the condensation fraction, transition temperature, and energy per particle for three-dimensional infinite homogeneous Bose gas using a potential model.

\section{Theoretical framework}

The static fluctuation approximation for neutral many-bosonic system will be present in this section for a potential model described by

$$
V(r)=\left\{\begin{array}{cc}
V_{0}, & r \leq a \\
0, & r>0,
\end{array}\right.
$$

where $V_{0}$ and $a$ are the constant potential strength and core radius.

The closed set of nonlinear integral equations describing any infinite homogeneous Bose system is $[2,3]$ :

$$
\hat{E}_{k}=\varepsilon(k)+\frac{1}{\Omega} \sum_{\boldsymbol{q}} W(\boldsymbol{k}, \boldsymbol{q}) \hat{n}_{q},
$$

where $\boldsymbol{k}$ is the linear momentum of the particle, $\hat{E}_{k}$ is the local-field operator, $\Omega$ - the normalization volume, $\varepsilon(k)$ incorporates the kinetic energy for a particle and chemical potential $\mu$ which is given by $\varepsilon(k)=\frac{\hbar^{2} k^{2}}{2 m}-\mu$, and 
$W(\boldsymbol{k}, \boldsymbol{q})$ represents the Fourier transform of the potential defined as $W(\boldsymbol{k}, \boldsymbol{q})=V(0)+V(|\boldsymbol{k}-\boldsymbol{q}|)$.

The occupation number is given by

$$
\begin{gathered}
n(k)=\frac{1}{2}\left[\frac{1}{\exp \left(\beta\left(\left\langle\hat{E}_{k}\right\rangle+\varphi_{k}\right)\right)-1}\right. \\
\left.\quad+\frac{1}{\exp \left(\beta\left(\left\langle\hat{E}_{k}\right\rangle-\varphi_{k}\right)\right)-1}\right],
\end{gathered}
$$

the fluctuation $\varphi_{k}$ in the local-field operator is determined from the following equation:

$$
\eta(k) \varphi_{k}^{2}=\frac{1}{\Omega} \sum_{\boldsymbol{p}} W(\boldsymbol{k}, \boldsymbol{p})\left\langle\Delta \hat{n}_{k} \Delta \hat{n}_{p}\right\rangle,
$$

the fluctuation is a function of the correlation function $\left\langle\Delta \hat{n}_{k} \Delta \hat{n}_{q}\right\rangle$, which is given by

$$
\left\langle\Delta \hat{n}_{k} \Delta \hat{n}_{q}\right\rangle_{c}=\frac{\eta(k)}{\Omega} \sum_{\boldsymbol{p}} W(\boldsymbol{k}, \boldsymbol{p})\left\langle\Delta \hat{n}_{p} \Delta \hat{n}_{q}\right\rangle
$$

and

$$
\begin{aligned}
& \left\langle\left(\Delta \hat{n}_{k}\right)^{2}\right\rangle=\left\langle\hat{n}_{k}\right\rangle\left(1+\left\langle\hat{n}_{k}\right\rangle\right) \\
& +\frac{2 \eta(k)}{\Omega} \sum_{\boldsymbol{p}} W(\boldsymbol{k}, \boldsymbol{p})\left\langle\Delta \hat{n}_{p} \Delta \hat{n}_{k}\right\rangle .
\end{aligned}
$$

The index $c$ in Eq. (4) denotes that $k \neq q$. The function $\eta(k)$ is given by

$$
\begin{gathered}
\eta(k)=\frac{1}{2 \varphi_{k}}\left[\frac{1}{\exp \left(\beta\left(\left\langle\hat{E}_{k}\right\rangle+\varphi_{k}\right)\right)-1}\right. \\
\left.-\frac{1}{\exp \left(\beta\left(\left\langle\hat{E}_{k}\right\rangle-\varphi_{k}\right)\right)-1}\right] .
\end{gathered}
$$

Finally, the chemical potential can be determined from the conservation of the total number of particles

$$
N=\sum_{\boldsymbol{k}}\left\langle\hat{n}_{k}\right\rangle \text {. }
$$

This set of nonlinear integral equations is solved numerically, where the mass $m$ is chosen to be the ${ }^{4} \mathrm{He}$ atomic mass and the density number is taken to be the liquid helium number density $n=0.02165 \AA^{-3}$. After that, the condensation fraction, critical temperature, and the energy per particle are calculated. Throughout our calculations a natural system of units has been used, such as $\hbar=1=m$, the conversion factor being $\frac{\hbar^{2}}{m}=12.120048 \mathrm{~K} \AA^{2}$.

\section{Results and discussion}

The closed system of nonlinear integral equations obtained in Sect. 2 is solved numerically over the potential strength ranges $V_{0}(0-25 \mathrm{~K})$ and core radius $a$ $(0.0-2.5 \AA)$. The transition temperature is calculated from the BEC fraction results. A remarkable phase transition in the energy per particle is observed.
Figure 1 shows the condensation fraction versus temperature at different values of potential strength. We observe that the BEC fraction and transition temperature depend strongly on the potential strength. As the potential strength increases, the condensation fraction decreases. The transition temperature, $T_{\mathrm{c}}$, is determined from Fig. 1, it is decreased by increasing the potential strength, as can be deduced from Table, this result is in consistence with results obtained in [5], Hartree-Fock theory calculation [12], and with renormalization group calculation [13]. As the potential strength increases, the equilibrium interparticle spacing increases and the probability of overlapping between the thermal waves decreases.

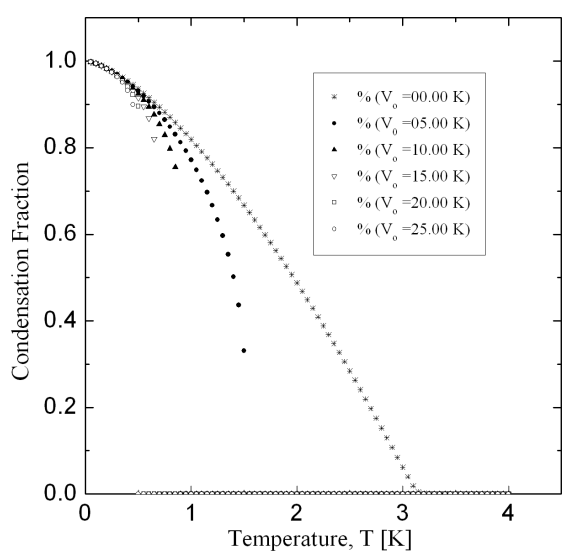

Fig. 1. The condensation fraction of Bose gas as a function of temperature $T$ for different values of potential strength $V_{0}$ at constant core radius $a=2.5 \AA$.

TABLE

The transition temperature of Bose gas for different values of potential strength $V_{0}$ at constant core radius $a=2.5 \AA$.

\begin{tabular}{l|c|c|c|c|c|c}
\hline \hline$V_{0}[\mathrm{~K}]$ & 00.00 & 05.00 & 10.00 & 15.00 & 20.00 & 25.00 \\
$T_{\mathrm{c}}[\mathrm{K}]$ & 3.14 & 1.55 & 0.90 & 0.70 & 0.55 & 0.50
\end{tabular}

The effect of the core radius was also studied at $V_{0}=10 \mathrm{~K}$. Figure 2 shows the condensation fraction versus temperature at different values of the core radius. It is clear that the transition temperature decreases as the core radius increases. As the core radius increases, the equilibrium interparticle spacing increases. This, in turn, decreases the condensation fraction. It is clear that the condensation fraction and transition temperature are strongly dependent on the potential parameters.

The effect of the potential parameters on the energy per particle has been studied. Figures 3 and 4 show the potential strength and the core radius effect versus temperature, respectively. Both figures show a discontinuity at temperatures equal to the transition temperature detected from the condensation fraction figure for interacting system. This discontinuity may represent a phase 


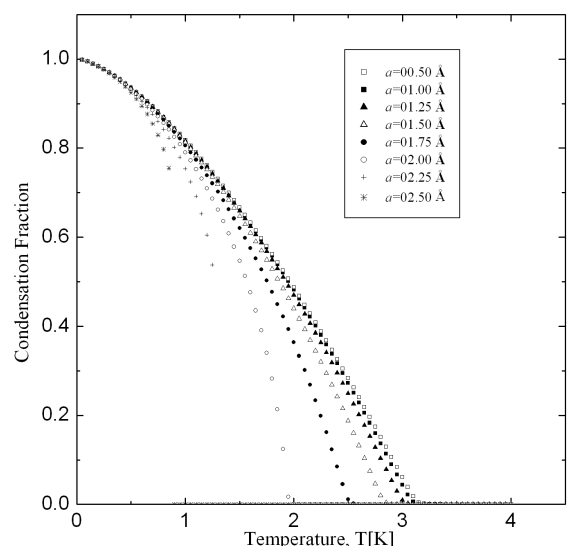

Fig. 2. The condensation fraction of Bose gas as a function of temperature $T$ for different values of core radius $a$ at constant potential strength $V_{0}=10 \mathrm{~K}$.

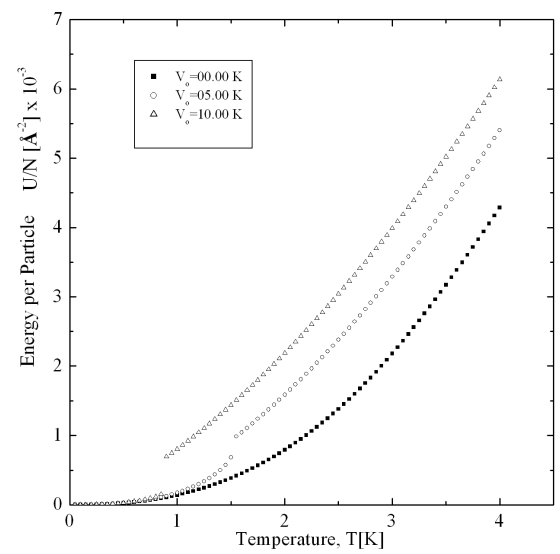

Fig. 3. The energy per particle of Bose gas as a function of temperature $T$ for different values of potential strength at constant core radius $a=2.5 \AA$.

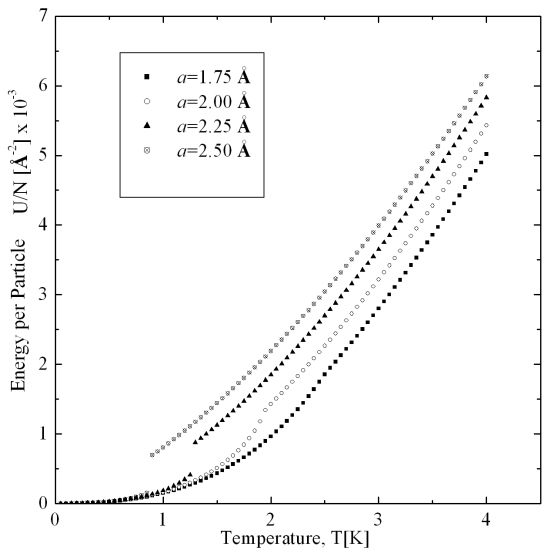

Fig. 4. The energy per particle of Bose gas as a function of temperature $T$ for different values of core radius $a$ at constant potential strength $V_{0}=10 \mathrm{~K}$. transition from superfluidity state to the normal state, which is in consistence with Ref. [6] for Bose systems in three dimensions, where both superfluidity and BEC occur at the same transition temperature.

At low temperatures, $T<T_{\mathrm{c}}$, the energy per particle is close to the corresponding value of the ideal system whatever the potential strength or core radius is. The effect of the potential parameters arises at $T>T_{\mathrm{c}}$, and the energy per particle is shifted up, as shown in Figs. 3 and 4 . The amount of shift increases with increasing the potential parameters. The discontinuity in energy per particle cannot appear precisely at small values of core radius.

\section{Conclusion}

$>$ From these results we may conclude that the potential strength effect appears more precisely in the physical quantities comparing with the core radius. At high density, namely liquid helium density, the condensation fraction and the transition temperature decrease with increasing the potential strength and/or core radius. Both BEC and superfluidity occur at the same transition temperature.

\section{References}

[1] M.H. Kalos, D. Levesque, L. Verlet, Phys. Rev. A 9, 2178 (1974).

[2] M.K. Al-Sugheir, H.B. Ghassib, R.R. Nigmatullin, Int. J. Theor. Phys. 40, 1033 (2001).

[3] B.R. Joudeh, M.K. Al-Sugheir, H.B. Ghassib, Int. J. Mod. Phys. B 19, 3987 (2005).

[4] A.S. Sandouqa, M.K. Al-Sugheir, H.B. Ghassib, Int. J. Theor. Phys. 45, 159 (2006).

[5] A. Griffin, Phys. Rev. B 53, 9341 (1996).

[6] P. Gruter, D. Ceperley, F. Laloe, Phys. Rev. Lett. 79 , 3549 (1997).

[7] K. Huang, Studies in Statistical Mechanics, Vol. II, North-Holland, Amsterdam 1964.

[8] H.T.C. Stoof, Phys. Rev. A 45, 8398 (1992).

[9] G. Baym, C.J. Pethick, Phys. Rev. Lett. 76, 6 (1996).

[10] C.C. Bradley, C.A. Sackett, J.J. Tollett, R.G. Hulet, Phys. Rev. Lett. 75, 1687 (1996).

[11] K.A. Brueckner, K. Sawada, Phys. Rev. 106, 1128 (1957).

[12] A.L. Fetter, J.D. Walecka, Quantum Theory of Many Particle System, McGraw-Hill, New York 1971.

[13] T. Toyoda, Ann. Phys. (New York) 141, 154 (1982). 